\title{
Tunable dual-wavelength operation of an all- fiber thulium-doped fiber laser based on tunable fiber Bragg gratings
}

\author{
B Ibarra-Escamilla ${ }^{1}$, M Durán-Sánchez ${ }^{2}, \mathrm{R}$ I Álvarez-Tamayo ${ }^{3}$, \\ B Posada-Ramírez ${ }^{1}, \mathrm{P}$ Prieto-Cortés ${ }^{4}$, E A Kuzin ${ }^{1}$, José L Cruz ${ }^{5}$ and \\ Miguel V Andrés 5 \\ ${ }^{1}$ Instituto Nacional de Astrofísica, Óptica y Electrónica, Optics Department, L. E. Erro 1, Puebla, México, 2824 \\ ${ }^{2}$ CONACyT - Instituto Nacional de Astrofísica, Óptica y Electrónica, Optics Department, L. E. Erro 1, \\ Puebla, México, 72824 \\ ${ }^{3}$ CONACyT-Universidad Autónoma de Nuevo León, Av. Universidad S/N, San Nicolás de los Garza, Nuevo León, México, 66451 \\ ${ }^{4}$ Universidad Autónoma de Nuevo León, Av. Universidad S/N, San Nicolás de los Garza, Nuevo León, México, 66451 \\ ${ }^{5}$ Applied Physics Department, Universidad de Valencia, ICMUV, Dr Moliner 50, E-46100 Burjassot, Spain
}

\begin{abstract}
Tunable dual-wavelength emission of a Tm-doped fiber laser based on two fiber Bragg gratings (FBGs) is experimentally demonstrated. By using two FBGs with central wavelengths at 2069.30 and $2069.44 \mathrm{~nm}$, stable dual-wavelength laser generation in the $2 \mu \mathrm{m}$ wavelength region is achieved by adjusting the differential loss of the two wavelengths in the laser cavity. Strain applied on the FBG allows independent tuning of the simultaneously generated wavelengths with separation between the laser lines in a range from 0.54 to $9 \mathrm{~nm}$. The laser has output power fluctuations less than $0.093 \%$ for an output power of $77.3 \mathrm{~mW}$.
\end{abstract}

Keywords: thulium-doped fiber, dual-wavelength fiber lasers, fiber bragg gratings, tunable fiber lasers

\section{Introduction}

Fiber lasers based on the use of a thulium-doped fiber (TDF) as a gain medium allow laser emission in the wavelength region from 1.8 to $2 \mu \mathrm{m}$. This operation feature makes TDF lasers (TDFL) attractive for applications in research areas such as gas detection, medicine, mechanical processing of polymers, light detection and ranging (LIDAR), and freespace optical communication [1-3]. Since fiber components operating in the $2 \mu \mathrm{m}$ wavelength region became readily accessible, different approaches of all-fiber TDFLs with stable dual-wavelength generation at the $2 \mu \mathrm{m}$ wavelength region were demonstrated. Wang et al [4] proposed a $2 \mu \mathrm{m}$ dual- wavelength fiber laser with coupled cavities based on fiber Bragg gratings (FBGs). Peng et al [5] employed two high birefringence (Hi-Bi) FBGs to achieve dual-wavelength laser emission of a $\mathrm{Tm}^{3+}$ fiber laser operating at $1.94 \mu \mathrm{m}$. Soltanian et al [6] reported stable dual-wavelength laser emission of a TDFL by using photonic crystal fibers acting as the infiber Mach Zehnder interferometer. However, achieving of stable dual-wavelength laser generation is challenging due to the strong mode competition caused by homogeneous broadening when TDF is used as gain medium. Different techniques have been proposed to reduce the competition based on the adjustment of intra-cavity losses. These techniques include the use of optical fiber devices such as 




Figure 1. Experimental setup of the tunable dual-wavelength TDFL.

polarization controllers (PCs) [7, 8], variable optical attenuators (VOAs) [9], optical fiber interferometers [10-13], and narrow bandwidth filters [14].

Moreover, the wide gain spectrum of the TDF makes TDFLs attractive for their application as widely tunable laser sources in optical instrumentation, microwave photonics, and terahertz generation at the $2 \mu \mathrm{m}$ region. Several methods to obtain tunable laser emission in TDFL have been reported, where the wavelength tuning of the generated laser line is based on different fiber devices such as Hi-Bi FBGs [14-20], tunable filters [21-23], fiber optical loop mirrors (FOLMs) [24, 25], and PCs [26-28]. In this regard, FBGs have proved their reliability as spectral filters for wavelength selection and tuning in fiber laser approaches. When FBGs are used as spectral filters, the laser emission line is tuned by applying strain on the FBG $[14,15]$. Double-cladding thulium-doped fiber (DCTDF) is attractive for design of high power and high beam quality fiber laser systems and fiber amplifiers operating at $2 \mu \mathrm{m}$ region. In conventional TDF, high beam quality is obtained at the expense of the output power due to the use of a single-mode pump in the core of the fiber. In contrast, DCTDFs are cladding pumped by a high-power multimodal pumping source allowing the increase of output power and the transformation of low-quality multimode pumping beams into single-mode laser emission with excellent quality of the output beams [29].

In this paper, we experimentally demonstrate a tunable dual-wavelength fiber laser operating in wavelengths over $2 \mu \mathrm{m}$. The ring cavity fiber laser configuration is based on the use of a DCTDF as gain medium. The simultaneously generated laser lines are independently tuned by using a pair of home-made tunable FBGs (TFBG) within the cavity in a novel configuration. The FBGs were designed to reach laser emission at wavelengths longer than the $2 \mu \mathrm{m}$. By strain application on the FBGs, dual-wavelength emission with tuned wavelength separation between the generated laser lines from $\sim 0.54$ to $\sim 9 \mathrm{~nm}$ is obtained. With the maximal wavelength separation the laser lines are generated at 2064.61 and $2073.63 \mathrm{~nm}$. The output power fluctuations of the generated laser lines in dual-wavelength operation for wavelength separations of 1.7 and $3.7 \mathrm{~nm}$ were less than $0.093 \%$.

\section{Experimental setup}

The laser consists of a ring cavity with fiber gratings connected in parallel as it is shown in figure 1. Three meters of a DCTDF are used as a gain medium. The DCTDF, provided by Coractive (DCF-TM-10/128), exhibit octagonal shaped pump inner cladding with the diameter of $128 \mu \mathrm{m}$, the numerical aperture of 0.45 , and the absorption of $4 \mathrm{~dB} \mathrm{~m}^{-1}$ at $790 \mathrm{~nm}$. The DCTDF is pumped by a multimode laser at $793 \mathrm{~nm}$ through a $(2+1) \times 1$ beam combiner (Lightcomm, China) used to combine pump and signal wavelengths at 793 and $2000 \mathrm{~nm}$, respectively. The beam combiner fiber pigtails have appropriated dimensions to minimize mismatch and losses in fiber splices. An optical isolator (ISO) is used to force unidirectional light propagation along the cavity. In order to obtain independent control of both generated laser

lines, an optical subsystem is formed with a 50/50 optical coupler. Each port of the coupler is connected to a TFBG through a VOA. The TFBGs are used as narrow band reflection spectral filters with maximal reflection at central wavelengths of $2069.30 \mathrm{~nm}$ for TFBG1 and $2069.44 \mathrm{~nm}$ for TFBG2. The FBGs are placed on a mechanical device which allows strain application on the FBGs by a micrometric screw displacement as it was reported in [24]. The VOAs were placed to independently adjust the cavity losses for each generated laser line by the TFBGs. The unconnected ends of the TFBGs (Output 1 and Output 2) are used for independently measurement of the TFBGs transmission and the generated laser lines. The $10 \%$ output port of a $90 / 10$ optical coupler (output 3 ) is used to measure simultaneously the laser wavelengths in the Output 3 . The output spectra are measured in each output (Outputs 1,2, and 3) by an optical spectrum analyzer (OSA, Yokogawa AQ6375) with a resolution of $0.05 \mathrm{~nm}$. 


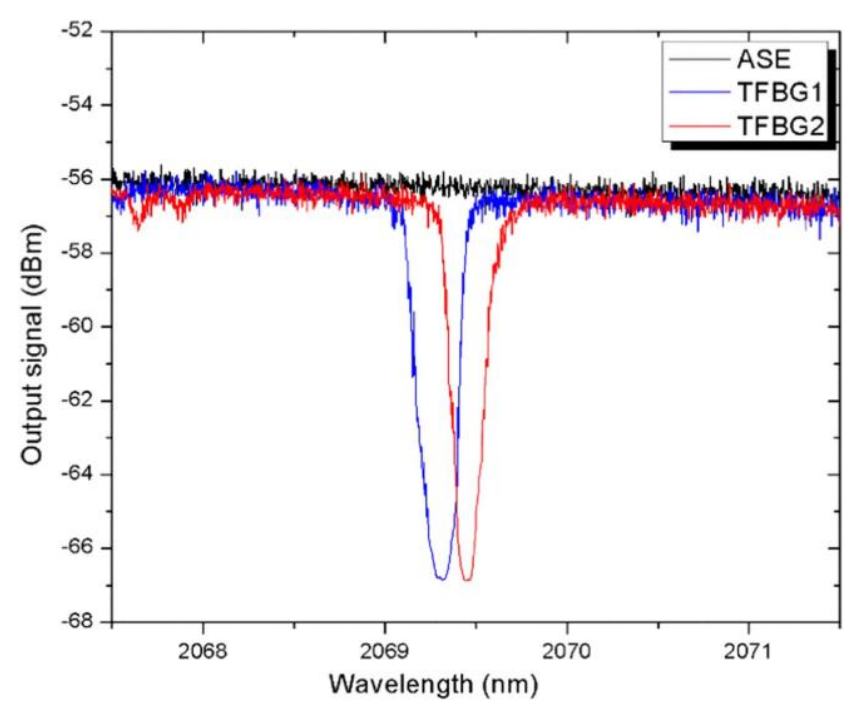

Figure 2. Transmission spectrum of the TFBGs for ASE of the DCTDF as input signal.

\section{Results and discussion}

Figure 2 shows the transmission spectrum of each TFBG at the central wavelength without strain application. In order to measure the transmission spectrum of the FBGs, the amplified spontaneous emission (ASE) of the DCTDF was used as input signal with pump power below the lasing threshold of $1 \mathrm{~W}$. The transmission spectrum of the TFBG1 and TFBG2 were measured at the Output 1 and the Output 2, respectively. The transmission spectra of both TFBGs were compared with the ASE spectrum of the DCTDF measured at the splice between the VOA and the TFBGs. The measurements were obtained without attenuation applied with the VOAs. As can be observed, the transmission spectra of the TFBGs exhibit transmission notches at the central wavelengths of $2069.30 \mathrm{~nm}$ and $2069.44 \mathrm{~nm}$ in which maximum reflection for TFBG1 (blue curve) and TFBG2 (red curve) are respectively obtained. The wavelength separation between the two central wavelengths is of $0.14 \mathrm{~nm}$. From a previous characterization of the TFBGs, the estimated attenuation at Bragg wavelength is of $\sim 25 \mathrm{~dB}$.

With the proposed configuration, dual-wavelength laser operation with equal powers can be achieved by adjusting the cavity losses by the use of the VOAs for the laser lines at the central wavelength corresponding to each TFBG. Figure 3 shows dual-wavelength laser generation of the TDFL with pump power of $2.5 \mathrm{~W}$. The output spectrum was measured at the output 3 . Figure 3(a) shows the simultaneously generated laser lines in dual-wavelength operation. Without strain application on the TFBGs, the cavity losses were adjusted by the use of the VOAs in order to achieve dual-wavelength laser emission with equal powers. As it can be observed, two laser lines at wavelengths $\lambda_{1}=2069.3$ and $\lambda_{2}=2069.44 \mathrm{~nm}$ are obtained. Figure 3(b) shows the single-wavelength laser emission measured at Output 2. As is shown, the shorter laser line of the dual-wavelength operation $\lambda_{1}$ corresponds to the wavelength filtered by the TFBG1. Likewise, the single laser wavelength $\lambda_{2}$ obtained at Output 1 corresponds to the laser wavelength generated by the TFBG2, as it is shown in figure 3(c). The full width at half maximum (FWHM) for both generated laser lines is measured as $0.05 \mathrm{~nm}$. This value is equal to the OSA resolution, so the FWHM of the lines is less or equal to $0.05 \mathrm{~nm}$.

Figure 4 shows the optical spectra of dual-wavelength operation of the TDFL. The measurements were obtained for different wavelength separation by application of stretching to FBG2 and compression to FBG1. The measurements were obtained at Output 3 with the OSA. As it can be observed in figure 4(a), the laser lines were initially tuned to 2068.98 and $2069.53 \mathrm{~nm}$ for minimum wavelength separation of 0.5 . Then, the generated laser lines were tuned to achieve a maximum wavelength separation of $9 \mathrm{~nm}$, as it is shown in figure 4(b). When micrometric displacement is applied in the center of the flexible metal sheet where the FBG is attached, the metal sheet is bent applying strain on the FBG. The FBG deformation leads to a displacement of the Bragg wavelength which allows the wavelength tuning of the generated laser line. When the linear displacement is applied on the side of the metal sheet where the FBG is attached, compression is applied on the FBG leading to a Bragg wavelength displacement to shorter wavelengths that can be linear fitted with a $-96 \mu \mathrm{mm}^{-1}$. On the other hand, when the FBG is stretched, Bragg wavelength displacement to longer wavelengths of $93.7 \mu \mathrm{m} \mathrm{m}^{-1}$, is observed [24]. In order to obtain dualwavelength with approximately equal output powers for each laser lines tuning, the cavity losses were adjusted by using the VOA in each arm of the TFBGs. As can be observed, the generated laser lines are wavelength tuned independently. The tuning range was limited to avoid damage of the TFBGs under stretching/compression; however, the wavelength range can be enlarged by improving the construction robustness of the TFBGs.

As mentioned above, by adjusting the VOAs in the two arms of the cavity, the TDFL can operate as tunable singlewavelength laser and also can be switched to operate as stable dual-wavelength laser. Figure 5 shows the stability of the dual-wavelength laser. The output spectrum stability of the dual-wavelength laser generation is shown in figure 5(a). A set of 25 laser output spectra measured each 2 min was obtained at room temperature. The results were obtained at Output 3 by the OSA with pump power of $2.5 \mathrm{~W}$. The laser lines were adjusted to generate output lasing at the wavelengths of 2069.00 and $2070.7 \mathrm{~nm}$. The data is presented in linear scale, as it can be observed, the dual-wavelength laser emission exhibit good stability. The proposed laser configuration allows carrying out a detailed study of the output power stability for each generated laser line. The output power fluctuations of each generated laser line in dualwavelength operation with equal output powers is shown in figures 5(b) and (c) for wavelength separations of 1.7 and $3.7 \mathrm{~nm}$, respectively. The independent output power fluctuations of the generated laser lines were measured by using thermal power meters at Outputs 1 and 2, and monitored by two consoles (Thorlabs-PM100D). The output power fluctuations were real-time recorded by software (Thorlabs Power 

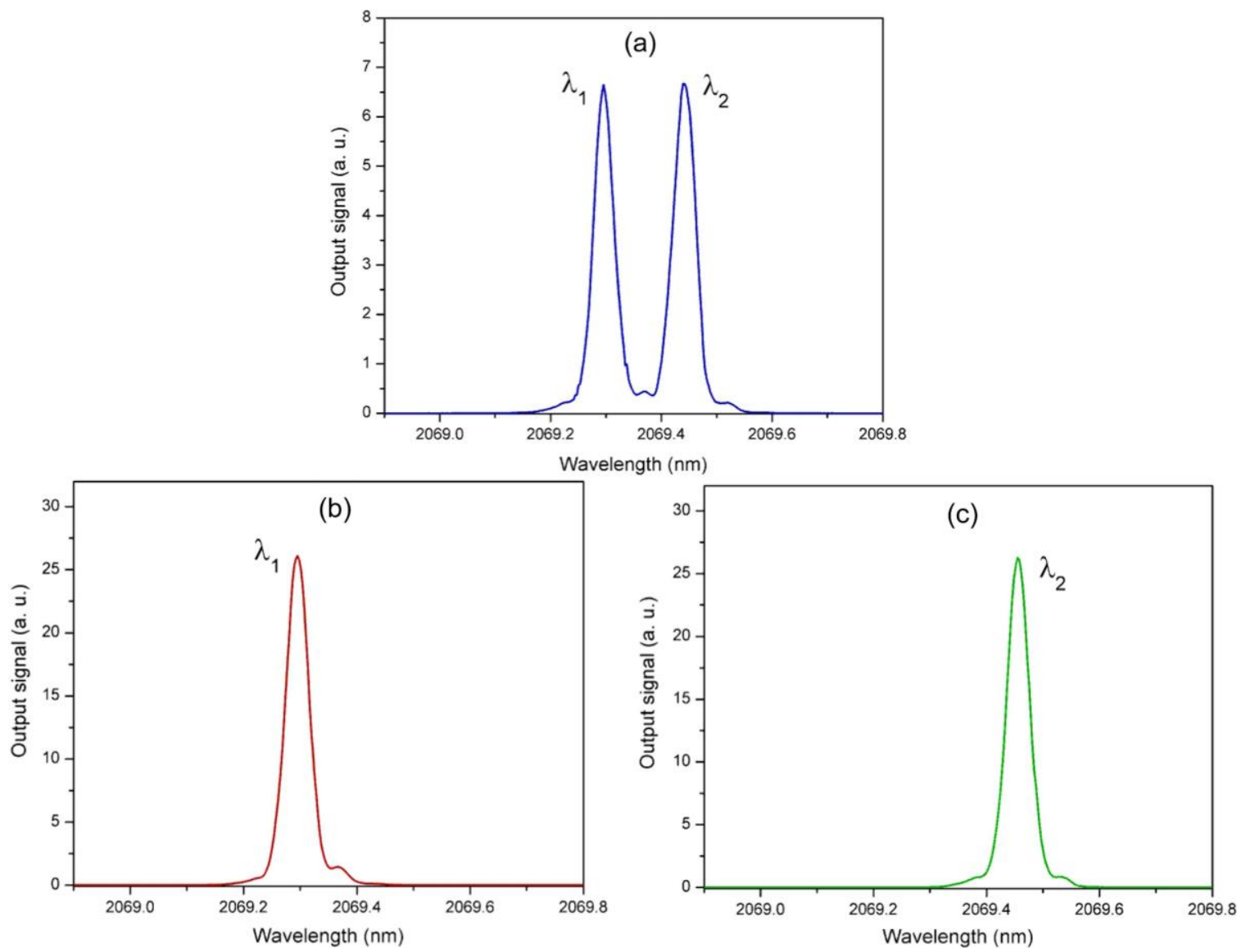

Figure 3. Laser spectrum of single and dual-wavelength operations. (a) Dual-wavelength 2069.30 and 2069.44 nm, single-wavelength: (b) $2069.30 \mathrm{~nm}$ and (c) $2069.44 \mathrm{~nm}$.
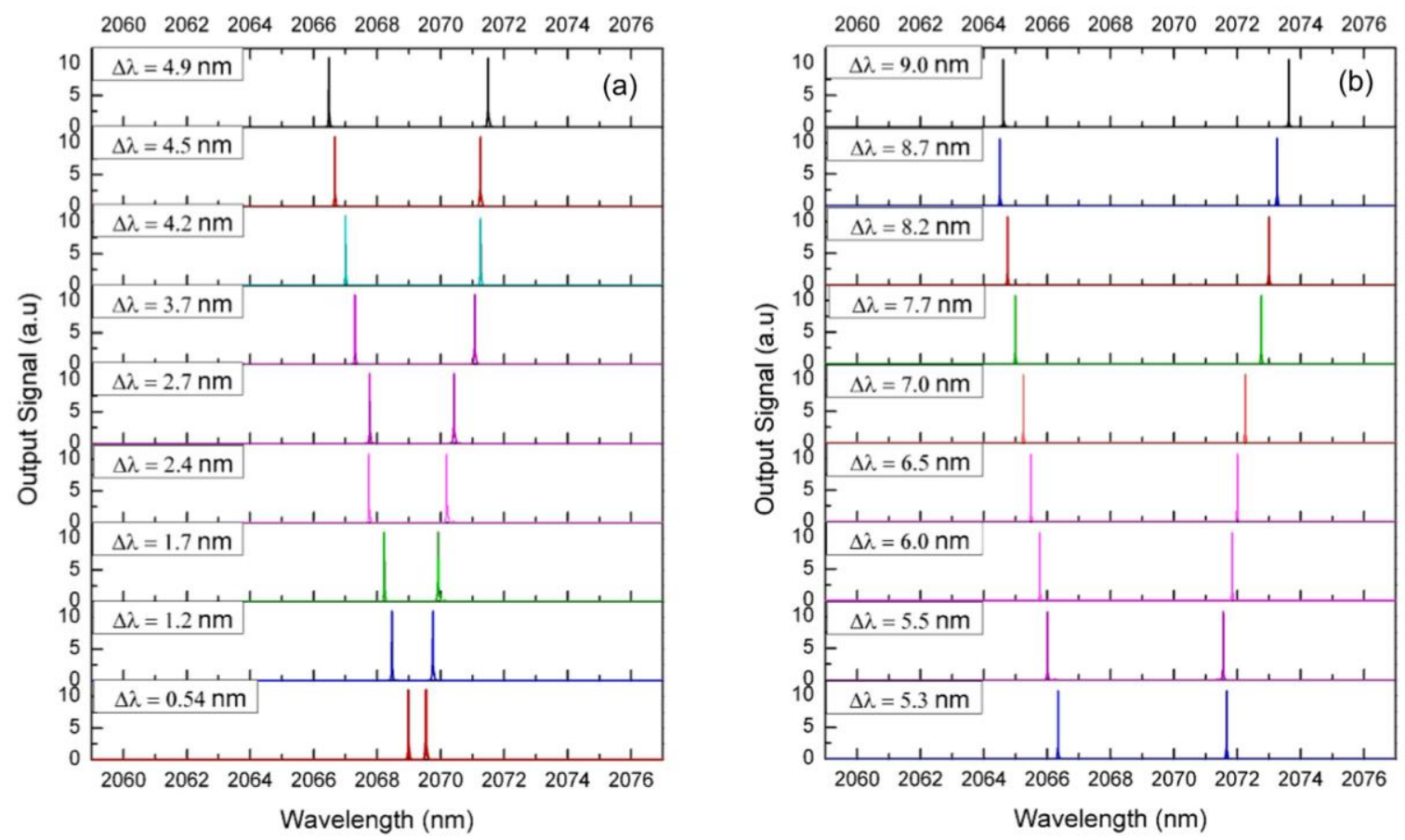

Figure 4. Tunable dual-wavelength laser operation with equal powers. 



Figure 5. (a) The output spectra stability of the laser in dual-wavelength operation. The continuously measured output power fluctuations of each generated laser line in dual-wavelength operation for wavelengths separation of: (b) $1.7 \mathrm{~nm}$, (c) $3.7 \mathrm{~nm}$.

Meter Utility Software). The output power fluctuations were time scanned over a period of $2.7 \mathrm{~h}$ at room temperature. As is depicted in figure $5(\mathrm{~b})$ for wavelengths separation of $1.7 \mathrm{~nm}$, the strong gain competition leads to an increase of the output power of $\lambda_{2}$ at $2069.92 \mathrm{~nm}$ as the output power of $\lambda_{1}$ at $2068.22 \mathrm{~nm}$ decreases and vice versa. The power fluctuations were computed from the standard deviation of the output power recorded data for each laser wavelength measured separately. Then, with the output power for $\lambda_{1}$ of $77.3 \mathrm{~mW}$, the fluctuation of the output power is within $0.093 \%$. Likewise, $\lambda_{2}$ with output power of $73.3 \mathrm{~mW}$, exhibits output power fluctuations within 0.073. In case of dual-wavelength generation at wavelengths $\lambda_{1}=2067.29$ of $\lambda_{2}=2071.07 \mathrm{~nm}$ with wavelengths separation of $3.7 \mathrm{~nm}$ shown in figure $5(\mathrm{c})$, the output power fluctuations for $\lambda_{1}$ and $\lambda_{2}$ with output powers of $70.3 \mathrm{~mW}$ and $66.2 \mathrm{~mW}$ are of $0.058 \%$ and $0.067 \%$, respectively.

The proposed dual-wavelength fiber laser can be used as single device in applications were multiple wavelengths are required [30]. In addition, the independent tunability of the simultaneously generated laser lines gives an additional degree of freedom that reduces the cost and complexity of systems having a large number of optical carriers, for instance, allows fast interrogation of self-referenced Bragg grating sensors [31] or to reduce by a factor of two the number of tunable lasers in continuous microwave phase shifters [32,33]. The detailed results demonstrate stability of the generated laser lines in dual-wavelength operation. However, different improvements can be taken into account in order to reduce the strong mode competition which leads to power fluctuation of the simultaneously generated laser lines. These techniques are based on the use of an optical device which allows the adjustment of the intra-cavity losses to reduce competition between the generated laser lines [14-28]. In our experience, we have demonstrated stable dual-wavelength laser emission of TDFL based on the use of a FOLM with Hi-Bi fiber loop [24, 25]. The use of the Hi-Bi FOLM allows the fine adjustment of the cavity losses between the generated laser lines with stability improvement. The proposed method has been demonstrated for fiber laser configurations which include the use of FBGs for selection and tuning of the generated laser lines [24].

\section{Conclusions}

In this paper, tunable and stable dual-wavelength laser generation of a ring cavity DCTDF laser was experimentally demonstrated. With a pair of TFBGs operating in the region of $2 \mu \mathrm{m}$ in a novel configuration, the wavelength of the 
generated laser lines are selected and independently tuned by strain application. The separation between the generated laser lines was tuned in a range from 0.54 to $9 \mathrm{~nm}$. By using a VOA for each TFBG, the cavity losses are adjusted in order to obtain stable dual-wavelength laser emission with equal out- put powers. For wavelength separations of 1.7 and $3.7 \mathrm{~nm}$ in dual-wavelength operation, the output power fluctuations of the generated laser lines were less than $0.093 \%$.

\section{Acknowledgments}

M Durán-Sánchez was supported by the CONACyT Grants 256401. M Durán-Sánchez and R I AlvarezTamayo want to thank to Cátedras CONACyT program. J L Cruz and M V Andrés acknowledge the Ministerio de Economía y Compe- titividad of Spain (MINECO) and Fonds Européen de Développement Économique et Régional (FEDER) (Grant TEC2016-76664-C2-1-R), and the Generalitat Valenciana (Grant PROMETEOII /2014/ 072).

\section{References}

[1] Pal A, Sen R, Bremer K, Yao S, Lewis E, Sun T and Grattan K T V 2012 All fiber tunable laser in the $2 \mu \mathrm{m}$ region, designed for $\mathrm{CO}_{2}$ detection Appl. Opt. 5। 7011-5

[2] Fried N M, Logada G A, Scott N J, Su L-M and Burnett A L 2008 Noncontact stimulation of the cavernous nerves in the rat prostate using a tunable-wavelength thulium fiber laser, J. Endourol. 22 409-13

[3] Mingareev I, Weirauch F, Olowinsky A, Shah L, Kadwani P and Richardson M 2012 Welding of polymers using a $2 \mu \mathrm{m}$ thulium fiber laser Opt. Laser Technol. 44 2095-9

[4] Wang Y, Zhou Y, Yan S, Tang Y and Xu J 2016 Dualwavelength $2 \mu \mathrm{m}$ fiber laser with coupled fiber Bragg grating cavities IEEE Photonics Technol. Lett. 28 1193-6

[5] Peng W J, Yan F P, Li Q, Liu S, Feng T, Tan S Y and Feng S C $20131.94 \mu \mathrm{m}$ switchable dual-wavelength Tm3 + fiber laser employing high-birefringence fiber Bragg grating Appl. Opt. 52 4601-7

[6] Soltanian M R K, Ahmad H, Khodaie A, Amiri I S, Ismail M F and Harun S W 2015 A stable dual-wavelength thulium-doped fiber laser at $1.9 \mu \mathrm{m}$ using photonic crystal fiber Sci. Rep. 514537

[7] Fan X, Zhou W, Wang S, Liu X, Wang Y and Shen D 2016 Compact dual-wavelength thulium-doped fiber laser employing a double-ring filter Appl. Opt. 55 3319-22

[8] Liu S, Yan F, Feng T, Wu B, Dong Z and Chang G K 2014 Switchable and spacing-tunable dual-wavelength thuliumdoped silica fiber laser based on a nonlinear amplifier loop mirror Appl. Opt. 53 5522-6

[9] Yang W, Lu P, Wang S, Liu D and Zhang J $20152 \mu \mathrm{m}$ switchable, tunable and power-controllable dual-wavelength fiber laser based on parallel cavities using $3 \times 3$ coupler Appl. Phys. B I20 349-54

[10] Álvarez-Tamayo R I, Duran-Sánchez M, Pottiez O,

Ibarra-Escamilla B, Cruz J L, Andres M V and Kuzin E A 2013 A dual-wavelength tunable laser with superimposed fiber Bragg gratings Laser Phys. 23055104

[11] Jia C, Liang X, Rochette M and Chen L R 2015 Alternate wavelength switching in a widely tunable dual-wavelength Tm3 + doped fiber laser at $1900 \mathrm{~nm}$ IEEE Photonics J. 7 1502907

[12] Ma X, Luo S and Chen D 2014 Switchable and tunable thulium doped fiber laser incorporating a Sagnac loop mirror Appl. Opt. 53 4382-5

[13] Liu S, Yan F, Liu P, Zhang L, Bai Z, Yin B and Zhou H 2016 Switchable single-polarization dual-wavelength dualwavelength TDFL using PM Fabry-Perot filter Opt. Fiber Technol. 29 1-5

[14] Liu S, Yan F, Peng W, Feng T, Dong Z and Chang G 2014 Tunable dual-wavelength thulium-doped fiber laser by employing a HB-FBG IEEE Photonics Technol. Lett. 26 1809-12

[15] Liu X, Han D, Sun Z, Zeng C, Lu H, Mao D, Cui Y and Wang F 2013 Versatile multi-wavelength ultrafast fiber laser mode-locked by carbon nanotubes Sci. Rep. 32718

[16] Liu X, Cui Y, Han D, Yao X and Sun Z 2015 Distributed ultrafast fibre laser Sci. Rep. 59101

[17] Han D D, Liu X M, Cui Y D, Wang G W, Zeng C and Yun L 2014 Simultaneous picosecond and femtosecond solitons delivered from a nanotube-mode-locked all-fiber laser $O p t$. Lett. 39 1565-8

[18] Zang Z 2013 All-optical switching in Sagnac loop mirror containing an ytterbium-doped fiber and fiber Bragg grating Appl. Opt. 52 5701-6

[19] Zang Z and Zhang Y 2012 Analysis of optical switching in a $\mathrm{Yb}^{3+}$-doped fiber Bragg grating by using self-phase modulation and cross-phase modulation Appl. Opt. 5 I 342430

[20] Zang Z G and Zhang Y J 2012 Low-switching power (<45 $\mathrm{mW}$ ) optical bistability based on optical nonlinearity of ytterbium-doped fiber with a fiber Bragg grating pair J. Mod. Opt. 59 161-5

[21] Wei Y, Hu K, Sun B and Wang T 2012 All-Fiber widely wavelength tunable thulium-doped fiber ring laser incorporating a Fabry-Perot filter Laser Phys. 22 770-3

[22] Ma X, Chen D, Shi Q, Feng G and Yang J 2014 Widely tunable thulium-doped fiber laser based on multimode interference with a large no-core fiber J. Lightwave Technol. 32 3234-8

[23] Hernández-Arriaga M V, Duran-Sánchez M, Ibarra-Escamilla B, Álvarez-Tamayo R I, Santiago-Hernández H, BelloJiménez M and Kuzin E A 2017 Tunable thulium-doped fiber laser based on an abrupt- tapered in-fiber interferometer $J$. Opt. 19115704

[24] Duran-Sánchez M, Álvarez-Tamayo R I, Posada-Ramirez B, Ibarra-Escamilla B, Kuzin E A, Cruz J L and Andrés M V 2017 Tunable dual-wavelength thulium-doped fiber laser based on FBGs and a Hi-Bi FOLM IEEE Photonics Technol. Lett. 29 1820-3

[25] Posada-Ramírez B, Duran-Sánchez M, Álvarez-Tamayo R I, Ibarra-Escamilla B, Bravo-Huerta E and Kuzin E A 2017 Study of a Hi-Bi FOLM for tunable and dual-wavelength operation of a thulium-doped fiber laser Opt. Express 25 2560-8

[26] He W, Zhu L, Dong M and Luo F 2016 Tunable and switchable thulium doped fiber laser utilizing Sagnac loops incorporating two-stage polarization maintaining fibers $\mathrm{Opt}$. Fiber Technol. 29 65-9

[27] Wang T, Miao X, Zhou X and Qian S 2012 Tunable multiwavelength fiber laser based on a double Sagnac Hi-Bi fiber loop Appl. Opt. 5I C111-6

[28] Ismail M F B, Dernaika M, Khodaei A, Harun S W and Ahmad H 2015 Tunable dual-wavelength thulium-doped fiber laser at $1.8 \mu \mathrm{m}$ region using spatial-mode beating $J$. Mod. Opt. 62 892-6

[29] Peterka P, Kubeček V, Dvořáček P, Kašík I and Matejec V 2006 Experimental demonstration of novel end-pumping method for double-clad fiber devices Opt. Lett. 3। 3240-2

[30] Walsh B M 2010 Dual-wavelength lasers Laser Phys. 20 622-34

[31] Mora J, Diez A, Cruz J L and Andrés M V 2000 A magnetostrictive sensor interrogated by fiber gratings for DC-current and temperature discrimination IEEE Photonics 
Technol. Lett. 12 1680-2

[32] Cruz J L, Ortega B, Andres M V, Gimeno B, Pastor D, Capmany J and Dong L 1997 Fibre Bragg gratings for phased-array antennas Electron. Lett. 33 545-6

[33] Huang S, Li J, Ye Y, Shi P, Zhou J, Guo B and Gu W 2012 The further investigation of the true time delay unit based on discrete fiber Bragg gratings Opt. Laser Technol. $44776-80$ 